\title{
Comparative Prospective Study Between Platlete Rich Plasma and Steroid Injection in Treatment of Chronic Planter Fasciitis
}

\author{
Abdel Rahman Abo takia-Abdel rahman El sheikh-Moustafaismail-Waelsalama \\ Department of orthopedics and Traumatology sohag university Egypt
}

\begin{abstract}
Purpose:Compare the results of injection of steroid and PRP in cases of chronic plantar fasciitis

INTRODUCTION: plantar fasciitis can be a difficult condition to treat. results of platelet rich plasma (PRP) injection have been promising. We compared PRP to cortisone injection in the treatment of chronic plantar fasciitis resistant to conservative management.
\end{abstract}

METHODS:38 heels (20 heels in the steroid group and 18heels in the PRP group) with plantar fasciitis failed conservative treatment were included to receive either PRP or Steroid injection. All patients were assessed by Visual Analogue Score (VAS) for pain, at 2 weeks 1 month and 3months post injection .

RESULTS:Our study included 38 patients; 20 in the steroid arm and 18 had PRP injections. The average age of the steroid injection group was $43.1 \pm 9.7$ years and in the PRP was $43.1 \pm 8.4$ years $(\mathrm{P}=0.698)$.

The starting average pain scores were 8.6 for both groups $(\mathrm{P}=0.712)$. Then our patients were followed up clinically at 2 weeks, 1 month and 3 months following the injections. There was statistically significant lower VAS scores for the steroid injection group at all follow up visits ( $\mathrm{P}<0.001$ in all follow ups). The average pain scores were 4.9 and 6.7 at 2 weeks follow up for the steroid injection versus the PRP respectively, it was 2 and 4.2 at 1 month and last 0.6 and 1.2 after 3 months respectively.

CONCLUSIONS:

This study demonstrates that both steroid and PRP injections are highly effective in treatment of chronic plantar fasciitis but improvement in pain was more rapid with steroid injection. This study recommends for follow up for a longer period than three months to compare long term benefits of steroids and PRP.

\section{Introduction}

Chronic plantar fasciitis is a common problem that affects sport participants as well as inactive middleaged individuals $[\underline{6}, \underline{19}]$. In general, the condition is self-limiting, and the majority of cases spontaneously resolve regardless of type of intervention received (including placebo) [4]. Increasing knowledge of the pathology has led to the widespread application of a large number of conservative treatments for recalcitrant plantar fasciitis [16], including physiotherapy, plantarfascia-stretching exercises []], icepacks, night splints, prefabricated and custom-made insert, shoe modification, nonsteroidal antiinflammatory drugs (NSAIDs) and extracorporeal shock-wave therapy (ESWT) when conventional physical therapy is not effective [23]. Although the

effect of ESWT remains controversial, reliable evidence supports the use of this approach for treating chronic 
plantar fasciitis $[\underline{12}, \underline{18}]$. However, adverse effects such as pain during treatment, soft-tissue damage (bleeding, hematoma, paresthesia), nausea, the need for peripheral nerve block and costs should be considered when proposing this procedure [26].

Recently, promising results were reported with the use of platelet-rich plasma (PRP) injections for treating muscle and tendon injuries and degeneration $[\underline{9}-\underline{13}, \underline{15}, \underline{21}]$. The rationale for using PRP is to increase tendon regenerative abilities with a high content of cytokines and cells, in hyperphysiologic doses, which should promote cellular chemotaxis, matrix synthesis, and proliferation [20]. Degranulation of the alpha granules in platelets releases many different growth factors that can play a role in tissue regeneration processes. PRP represents a treatment option for many foot and ankle pathologies, including tendinopathy (Achilles, peroneal, posterior tibial, flexor hallucis longus, anterior tibial) and chronic ligamentous injury, such as plantar fasciitis.

The purpose of this study was to assess the safety of PRP injections for treating chronic plantar fasciitis and provide initial clinical assessment of its effectiveness.

\section{Material and methods}

38 consecutive patients ( 25 women, 13 men; mean age $43.2 \pm 8.8$ years) admitted to our hospital in 2016 were enrolled in this study. All patients gave informed consent to participate in the study, which was carried out in sohag university hospital. Patients were included if they were $\geq 18$ years, experienced heel pain felt maximally over the plantar aspect for at least six months continuously. Patients were treated in the prior three months with conservative therapies, such as icepacks, stretching of the Achilles tendon and NSAID medication, which provided inadequate improvement of pain and functionality. Exclusion criteria included generalised inflammatory arthritis, including ankylosing spondylitis, rheumatoid arthritis or psoriatic arthritis; any wound or skin lesion at the plantar aspect of the foot; pregnancy; severe infection; known malignancy; previous surgery, ESWT or corticosteroid injection into the heel, including Achilles tendon; nerve-related symptoms such as radiculopathy, tarsal tunnel syndrome or tarsi sinus syndrome; foot and ankle osteoarthritis.

Ten millilitres of autologous blood was taken from the antecubital vein with the outer syringe and placed into the Arthrex Centrifuge and centrifuged for five minutes at 1,500 rpm. During the extracorporeal blood processing, $2 \mathrm{ml}$ of anticoagulant citrate dextrose solution was used to prevent clotting. The system allows supernatant (PRP) transfer from the 10-ml outer syringe into the 5-ml syringe under aseptic conditions. All patients received one injection at the plantar fascia. injection was performed by one of the authors (SC) on an outpatient basis. The injection point was at the origin of the plantar fascia on the medial tubercle of the calcaneus, as described by Cyriax and Cyriax [5]. The origin of the plantar fascia was approached from the medial side of the foot but near the plantar surface. After injection, all patients were allowed to immediately walk but were advised to avoid weight-bearing sport activities, such as running or jumping, for at least four weeks after the last injection. After PRP injection, patients remained in the outpatient clinic until pain was considered tolerable and were followed in the outpatient clinic at 2 weeks 1 month and three months intervals or by telephone interview to detect possible side effects. NSAIDs were prescribed 
for no more than three days after injection, and ice packs were allowed for postinjection pain. Physiotherapy treatments were not prescribed during recovery from the injections.

Before treatment and during the follow-up visit, patients were asked to rate their pain on a visual analogue scale (VAS), with zero indicating no pain and ten the worst pain imaginable. $[14,24]$ was used to define the outcome of the procedure. Treatment was considered successful when the patient had an excellent or good score. Patients were examined clinically at 2 weeks after the index procedure. Data were analyzed using SPSS 13.0 (SPSS Inc., Chicago, IL, USA), with a paired $t$ test after checking normal distribution of samples with the Kolmogorov-Smirnoff $z$ test. The level of significance was considered as $p<0.0$

\section{Results}

Our study included 38 patients; 20 in the steroid arm and 18 had PRP injections. The average age of the steroid injection group was $43.1 \pm 9.7$ years and in the PRP was $43.1 \pm 8.4$ years $(\mathrm{P}=0.698)$.

There were 13 males and 25 females. The steroid injection group had 7 males and 13 females while the PRP group had 6 males and 12 females as shown in table 4.

Eleven right feet were injected in both the steroid and the PRP groups and 9 and 7 left feet were injected by steroid and PRP respectively as shown in table5.

Pain was assessed using VAS. The starting average pain scores were 8.6 for both groups $(\mathrm{P}=0.712)$. Then our patients were followed up clinically at 2 weeks, 1 month and 3 months following the injections.

There was statistically significant lower VAS scores for the steroid injection group at all follow up visits ( $\mathrm{P}<0.001$ in all follow ups). After 2 weeks of injection. The average pain scores were 4.9 and 6.7 for the steroid injection versus the PRP respectively, it was 2 and 4.2 at 1 month and last 0.6 and 1.2 after 3 months Go to The starting average pain scores were 8.6 for both groups $(\mathrm{P}=0.712)$. Then our patients were followed up clinically at 2 weeks, 1 month and 3 months following the injections. There was statistically significant lower VAS scores for the steroid injection group at all follow up visits ( $\mathrm{P}<0.001$ in all follow ups). The average pain scores were 4.9 and 6.7 at 2 weeks follow up for the steroid injection versus the PRP respectively, it was 2 and 4.2 at 1 month and last 0.6 and 1.2 after 3 months respectively.

\section{Discussion}

Our results confirmed by results of Say et al. (2014) who found a positive effect on pain and functional scores in the steroid group which can be explained by the anti-inflammatory effect. However, steroid injections have been reported to be related to plantar fascia tear, fat pad atrophy, abscess, and osteomyelitis (Buccilli et al. 2005). They concluded that he PRP group had significantly higher mean VAS scores at follow-up than the steroid group $(\mathrm{p}<0.001)$ which was similar to our results (Say et al. 2014).Also Yaratapalli et al. (2015) found that in the PRP and corticosteroid injection groups at the initial visit had VAS of 6.85 and 6.95 respectively. On

injection of PRP and corticosteroid in respective groups, 4 weeks evaluation of VAS showed a significant decrease in corticosteroid group as compared to PRP group. At the end of 8 weeks the VAS decreased significantly in corticosteroid group compared to PRP 
group. At the end of 3 months, the VAS decreased significantly in corticosteroid group as compared to PRP group. At the end of 6 months, the PRP group showed significant reduction in VAS compared to corticosteroid group. This shows that corticosteroid is more effective for short

term relief and PRP is more effective for long term relief (Yaratapalli et al. 2015).

Similar to our results, Lee and Ahmad. (2007) conducted prospective randomised, controlled study of 64 patients for a period of 6 months by comparing PRP with corticosteroid injection. The authors found that there is significant reduction in VAS for both the groups over a time. At 6 weeks and 3 months, the corticosteroid group had significantly lower VAS than the PRP group, but the difference was not significant at 6 months but in our study, we found a significant reduction in VAS score at 2 weeks, 1 months and 3 months with corticosteroid group.

Also Homayouni et al. (2016) study revealed that local injection of PRP furnishes consequential relief of pain and improvement in function that is comparable to the corticosteroid injection to treat PF. Corticosteroid injection in $\mathrm{PF}$, when conservative management is unsuccessful, is an effective treatment (Genc et al. 2005, Porter and Shadbolt. 2005). But some authors concluded that corticosteroid injection can give short-term relief and seems to be useful only to a small degree apparently, since intrafascial injection may lead to permanent adverse

changes within the fascial structure and since patients tend to overuse the foot after injection as a result of direct pain alleviation, fascial rupture is the side effect of repeated corticosteroids injections (Kim et al. 2010).
Aksahin et al. (2012) in their prospective, randomized controlled trial compared corticosteroid and PRP injections to treat PF, they reported that both methods impressively treated PF. Shetty et al. (2014) studied 60 patients and demonstrated the positive effect of PRP on PF after three months. Their study described the comparison of an autologous platelet concentrate injection with corticosteroid injection in patients with unsuccessful nonoperative treatment of PF. It exhibited that a single injection of autologous concentrated platelets decreased pain and improved function more than corticosteroid injection after three months. These improvements were sustained over time and complications were not reported. Ragab and Othman. (2012) reported a $60 \%$ success rate with PRP in patients with $\mathrm{PF}$ in three months follow-up, which were in contrast to our results.Also Martinelli et al. (2012) results of their series showed that three PRP injections provided improvement in VAS for pain, with symptom resolution in 78.6 $\%$ of the patients. This confirms reports by other authors that suggest an improved healing process of tendons following local administration of growth factors through PRP injections (Bosch et al. 2010, Andia et al. 2010).

\section{References}

1. Dunn JE, Link CL, Felson DT et al. Prevalence of foot and ankle conditions ina multiethnic community sample of older adults. Am J Epidemiol 2004; 159:491-498.

2. Riddle DL, Schappert SM. Volume of ambulatory care visits and patterns ofcare for patients diagnosed with plantar fasciitis: a national study of medicaldoctors. Foot Ankle Int 2004; 25: 303-310.

3. Riddle DL, Pulisic M, Pidcoe P, Johnson RE. Risk factors for plantar fasciitis: amatched case-control study. J 
Bone Joint Surg Am 2003; 85: 872877.

4. Gill LH. Plantar fasciitis: diagnosis and conservative management. J Am AcadOrthopSurg 1997; 5: 109-117.

5. Hicks JH. The mechanics of the foot. II. The plantar aponeurosis and the arch.J Anat 1954; 88: 25-30.

6. Woolnough J. Tennis heel. Med J Aust 1954; 2: 857.

7. Lemont $\mathrm{H}$, Ammirati KM, Usen $\mathrm{N}$. Plantar fasciitis: a degenerative process(fasciosis) without inflammation. J Am Podiatr Med Assoc 2003; 93: 234-237.

8. Schon LC, Glennon TP, Baxter DE. Heel pain syndrome: electrodiagnosticsupport for nerve entrapment. Foot Ankle 1993; 14: 129135.

9. Tanz SS. Heel pain. ClinOrthopRelat Res 1963; 28: 169-178.

10. Shmokler RL, Bravo AA, Lynch FR, Newman LM. A new use of instrumentation in fluoroscopy controlled heel spur surgery. J Am Podiatr Med Assoc 1988; 78:194-197.

11. Chimutengwende-Gordon $\mathrm{M}$, O'Donnell P, Singh D. Magnetic resonance imagingin plantar heel pain. Foot Ankle Int 2010; 31: 865-870.

12. Donley BG, Moore $\mathrm{T}$, Sferra $\mathrm{J}$ et al. The efficacy of oral nonsteroidal antiinflammatory medication (NSAID) in the treatment of plantar fasciitis: arandomized, prospective, placebocontrolled study. Foot Ankle Int 2007; 2820-23

13. Crawford F, Atkins D, Young P, Edwards J. Steroid injection for heelpain: evidence of short-term effectiveness. A randomized controlled trial.Rheumatology 1999; 38: 974-977.

14. Acevedo JI, Beskin JL. Complications of plantar fascia rupture associated withcorticosteroid injection. Foot Ankle Int 1998; 19: 91-97.
15. Leach R, Jones R, Silva T. Rupture of the plantar fascia in athletes. Bone JointSurg Am 1978; 60: 537-539.

16. DiGiovanni BF, Nawoczenski DA, Lintal ME et al. Tissue-specific plantar fasciastretchingexercise enhances outcomes in patients with chronic heel pain. Aprospective, randomized study. J Bone Joint Surg Am 2003; 85: 1,2701,277 .

17. DiGiovanni BF, Nawoczenski DA, Malay DP et al. Plantar fasciaspecificstretching exercise improves outcomes in patients with chronic plantar fasciitis.A prospective clinical trial with two-year follow-up. J Bone Joint Surg Am 2006;88: 1,775-1,778.

18. Wolgin M, Cook C, Graham C, Mauldin D. Conservative treatment of plantarheel pain: long-term follow-up. Foot Ankle Int 1994; 15: 97-102.

19. Snook GA, Chrisman OD. The management of subcalcaneal pain. ClinOrthopRelat Res 1972; 82: 163168.

20. Furey JG. Plantar fasciitis. The painful heel syndrome. J Bone Joint Surg Am 1975; 57: 672-673.

21. Babcock MS, Foster L, Pasquina P, Jabbari B. Treatment of pain attributed toplantar fasciitis with botulinum toxin a: a short-term, randomized, placebocontrolled,double-blind study. Am J Phys Med Rehabil 2005; 84: 649-654.

22. Ogden JA, Alvarez RG, Levitt RL et al. Electrohydraulic high-energy shockwavetreatment for chronic plantar fasciitis. J Bone Joint Surg Am 2004; $86: 2,216-2,228$.

23. Hammer DS, Rupp S, Kreutz A et al. Extracorporeal shockwave therapy (ESWT)

in patients with chronic proximal plantar fasciitis. Foot Ankle Int 2002; 23:309313.

24. Wang CJ, Chen HS, Huang TW. Shockwave therapy for patients with plantarfasciitis: a one-year follow-up 
study. Foot Ankle Int 2002; 23: 204 207.

25. Gerdesmeyer L, Frey C, Vester J et al. Radial extracorporeal shock wave therapyis safe and effective in the treatment of chronic recalcitrant plantar fasciitis:results of a confirmatory randomized placebo-controlled multicenter study. AmJ Sports Med 2008; 36: 2,100-2,109.

26. Anderson RB, Foster MD. Operative treatment of subcalcaneal pain. Foot Ankle1989; 9: 317-323.

27. Murphy GA, Pneumaticos SG, Kamaric E et al. Biomechanical consequences ofsequential plantar fascia release. Foot Ankle Int 1998; 19: 149-152.

28. Baxter DE, Pfeffer GB. Treatment of chronic heel pain by surgical release ofthe first branch of the lateral plantar nerve. ClinOrthopRelat Res 1992; 279:229-236.

29. Barrett SL, Day SV, Pignetti TT, Egly BR. Endoscopic heel anatomy: analysis of 200 fresh frozen specimens. J Foot Ankle Surg 1995; 34: 51-56.

30. Marafk C. Endoscopic partial plantar fasciotomy as a treatment alternative inplantar fasciitis. ActaChirOrthopTraumatolCech 2007; 74: 406-409.

31. Hogan KA, Webb D, Shereff M. Endoscopic plantar fascia release. Foot Ankle 